Article

\title{
Optimization and Application for Hydraulic Electric Hybrid Vehicle
}

\author{
Hsiu-Ying Hwang ${ }^{1}$, Tian-Syung Lan ${ }^{2, *}$ and Jia-Shiun Chen ${ }^{1}$ \\ 1 Department of Vehicle Engineering, National Taipei University of Technology, Taipei 10608, Taiwan; \\ hhwang@mail.ntut.edu.tw (H.-Y.H.); chenjs@mail.ntut.edu.tw (J.-S.C.) \\ 2 College of Mechatronic Engineering, Guangdong University of Petrochemical Technology, \\ Maoming 525000, China \\ * Correspondence: tslan888@gmail.com
}

Received: 27 November 2019; Accepted: 7 January 2020; Published: 9 January 2020

\begin{abstract}
Targeting the application of medium and heavy vehicles, a hydraulic electric hybrid vehicle (HEHV) was designed, and its energy management control strategy is discussed in this paper. Matlab/Simulink was applied to establish the pure electric vehicle and HEHV models, and backward simulation was adopted for the simulation, to get the variation of torque and battery state of charge (SOC) through New York City Cycle of the US Environmental Protection Agency (EPA NYCC). Based on the simulation, the energy management strategy was designed. In this research, the rule-based control strategy was implemented as the energy distribution management strategy first, and then the genetic algorithm was utilized to conduct global optimization strategy analysis. The results from the genetic algorithm were employed to modify the rule-based control strategy to improve the electricity economic performance of the vehicle. The simulation results show that the electricity economic performance of the designed hydraulic hybrid vehicle was improved by $36.51 \%$ compared to that of a pure electric vehicle. The performance of energy consumption after genetic algorithm optimization was improved by $43.65 \%$.
\end{abstract}

Keywords: hydraulic hybrid vehicle; NYCC driving cycle; optimization; genetic algorithm

\section{Introduction}

The increasing demand for fossil fuels in different fields since the Industrial Revolution has led to increasing global $\mathrm{CO}_{2}$ emission and worsening global warming. Among all $\mathrm{CO}_{2}$ emission, the emission of means of transportation is only second to the industry. Now, the passenger vehicles all develop toward alternative energy, whereas the medium and heavy vehicles for goods transportation are still using gasoline or diesel engines as the main power source. With global warming and increasing stringent laws and regulations, they will definitely develop toward the same clean energy as the passenger vehicles. According to Navigant Research, the market survey company, hydraulic hybrid vehicles seldom known and underestimated in significance will gain a position in the heavy-duty truck market, and even can be expected to apply to the next generation of vehicles. Therefore, hydraulic electric hybrid vehicles (HEHV) will be the first choice for medium vehicles, heavy vehicles, and common carriers. With the DSHplus software simulation, Sokar [1] compared the fuel economy of the hydraulic transmission vehicles and hydraulic hybrid vehicles in urban and highway driving cycles. Chen [2] compared the energy consumption of different hydraulic hybrid configurations, and it showed the HEHV could have better energy efficiency over the pure EV system. The energy optimization can be divided to hardware optimization and control strategy optimization. As for hardware optimization, Ramakrishnan et al. [3] proposed the study on influence of system parameters in hydraulic system on the overall system power and established the series hydraulic hybrid power 
vehicle with LMS AMESim software. Change of size of accumulator and hydraulic motor/pump and internal pressure greatly improves the output power of the whole system, which also reduces the fuel consumption and pollution of the hydraulic hybrid vehicles. The energy control strategy can be divided into two categories [4]: (1) rule-based strategy and (2) optimization-based strategy. For optimization strategy, Lu et al. [5] introduced the weighted-sum method and no-preference method to solve the multi-objective optimization problem of plug-in electric vehicles, and it was validated with ADVISOR software. Zeng et al. [6] proposed a different strategy, Equivalent Consumption Minimum Strategy (ECMS), to solve the optimization problem of PHEV, and the Simplified-ECMS strategy could effectively shorten the calculation time. Wang et al. [7] applied the Dynamic Programming for PHEV and received an approximately $20 \%$ improvement in fuel economy.

The rule-based control, featuring a smaller amount of calculation, is adopted by many studies, to design the energy management strategy. Yu et al. [8] developed a simulation model and rule-based control strategy for extended-range electric vehicle (E-REV) and showed that a small engine can be used to reduce the weight of vehicle and batteries of E-REV. Gao et al. [9] proposed two control strategies, thermostat and power follower. With dynamic programming, it showed that the thermostat control strategy optimized the operation of the internal combustion engine, and the power follower control strategy minimizes the battery-charging and-discharging operations. Konev et al. [10] developed a control strategy for series hybrid vehicle. The control strategy was to ensure gradual operation of the motor along the steady-state Optimal Operating Points Line (OOP-Line) in the engine speed-torque map, which could improve the efficiency of series hybrid vehicle. Liu et al. [11] developed a control strategy for a series hybrid vehicle which included two parts, constant SOC control, and driving-range optimization. Comparing to thermostat control strategy, the constant SOC control could have a longer driving range. Li et al. [12] proposed a fuzzy logic energy-management system, using the battery working state, which ensured that the engine would operate in the vicinity of its maximum fuel-efficiency region. The rule-based design is fast and easy and can be readily applied to real vehicle-control strategy. However, the rule-based control strategy is simple, so it cannot provide optimal power management to HEV in real time. Therefore, an optimization algorithm is required for rule-based control to improve the energy efficiency. Ho and Klong [13] introduced an optimization algorithm for series plug-in hybrid electric vehicles by utilizing the genetic algorithm (GA), which could determine the optimal driving patterns offline. Xu et al. [14] developed a fuzzy control strategy for parallel hybrid electric vehicle. The control strategy was adjusted with GA. It was verified that GA could effectively improve the efficiency of the engine and fuel consumption. Kaur et al. [15] proposed a control strategy to control the speed of a hybrid electric vehicle. The controller, which was using GA, could improve fuel economy and reduce pollution. Hu and Zhao [16] applied an adaptive based hybrid genetic algorithm to optimize the energy efficiency of parallel hybrid electric vehicles and presented the effectiveness of the hybrid genetic algorithm.

Therefore, global optimization, together with rule-based control method, are selected in this paper for medium and heavy vehicles in fixed driving route, to adjust the rule-based control strategy and improve the electricity economic performance of vehicles. The optimization approach selected in this paper is genetic algorithm (GA). With global optimization ability and probability optimization approach, GA can automatically obtain and instruct the optimized search space and adaptively adjust the search direction without the need of clear rules.

\section{Modeling}

In this study, Matlab/Simulink serves as the main simulation program, and backward simulation is used to establish the model. In order to compare the difference between an HEHV and a pure electric vehicle, subsystem models of the electric system are established, including models of electric motor, generator, and lithium ion battery. The subsystem models of hydraulic system include variable hydraulic motor/pump and accumulator models. The whole vehicle model can be divided into following subsystem models: (1) driver model; (2) vehicle dynamic model; (3) tyre and drive model; 
(4) power component element; and (5) energy storage component model. Driving cycle of the EPA NYCC is employed in this study to get the vehicle driving force, and then gear ratio of the transmission system is adopted to calculate the torque and speed needed for the motor. In HEHV, the electric motor does not function as the regenerative brake; rather the hydraulic pump is used for energy recovery. This is introduced in the following.

\subsection{Driver Model}

The EPA NYCC driving cycle for testing, as shown in Figure 1, is employed in this model. The total driving time is $599 \mathrm{~s}$. The stop time accounts for $35.08 \%$ of the total driving time. The maximum speed and the average speed are 44.6 and $11.4 \mathrm{~km} / \mathrm{h}$, respectively.

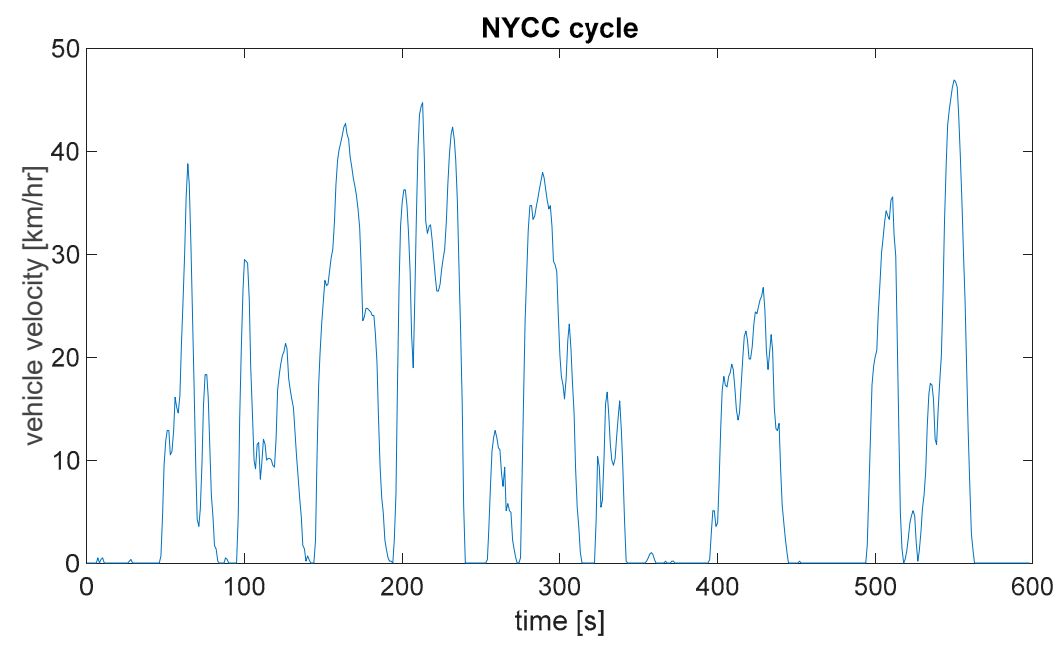

Figure 1. United States Environmental Protection Agency New York City Cycle (US EPA NYCC) driving cycle.

\subsection{Vehicle Dynamic Model}

A vehicle dynamic model is applied to respond to the driving tractive effort and resistance needed for the simulation vehicle. The resistance included rolling resistance $\left(R_{r}\right)$, aerodynamic resistance $\left(R_{a}\right)$, grading resistance $\left(R_{c}\right)$, and acceleration resistance $R_{s}$. The tractive effort for driving needed by the vehicle can be obtained with a vehicle dynamic model, which can be represented by Equation (1). The detailed calculation of resistance will be introduced in the following:

$$
F_{t}=R_{r}+R_{a}+R_{c}+R_{s}
$$

\subsubsection{Rolling Resistance}

During vehicle traveling, interaction force is produced in both radial and axial directions in the area where the wheels make contact with the ground, and there is also deformation between the tyre and the ground. The deformation process will be accompanied by a certain energy loss, regardless of whether or not it is in tyre or ground. This energy loss is the cause of rolling resistance during wheel turning. The rolling resistance can be represented by Equation (2), where $\mu_{r}$ is the rolling resistance coefficient, and $W$ is the vehicle weight:

$$
R_{r}=R_{r, A}+R_{r, B}=\mu_{r} \cdot W
$$

\subsubsection{Aerodynamic Resistance}

The aerodynamic resistance can be represented by Equation (3) as follows, where $C_{D}$ is the aerodynamic resistance coefficient, $\rho$ is the air density, $A_{f}$ is the front area of the vehicle, $v$ is the vehicle speed, and $v_{w}$ is the wind speed. 


$$
R_{a}=C_{D} \cdot \frac{\rho}{2} \cdot A_{f} \cdot\left(v-v_{w}\right)^{2}
$$

\subsubsection{Grading Resistance}

During climbing, grading resistance is produced due to the influence of the vehicle weight. During downhill, this resistance becomes the driving force instead. It can be represented by Equation (4), where $\theta$ is the slope angle:

$$
R_{c}=W \sin (\theta)
$$

\subsubsection{Acceleration Resistance}

The vehicle driving state covers the acceleration and deceleration for most of the time, except on highways, where it is fixed-speed driving. The required force for acceleration can be represented by Equation (5), where $a$ is the acceleration, and $g$ is the gravity acceleration:

$$
R_{s}=W \cdot \frac{a}{g}
$$

\subsection{Tyre and Drive Model}

Vehicle dynamics is used to analyze the vehicle tyre model. The angular speed $\left(\omega_{\text {drive }}\right)$ and the torque ( $T_{\text {drive }}$ ) of its transmission shaft can be represented by Equations (6) and (7), where GR is the final transmission gear ratio, $r$ is the tyre radius, $\eta_{f d}$ is the transmission efficiency, and $F_{\text {tire }}$ is the tyre force.

$$
\begin{aligned}
& \omega_{\text {drive }}=G R \cdot \frac{60}{2 \pi \cdot r} \cdot v \\
& T_{\text {drive }}=F_{\text {tire }} \cdot r \cdot \frac{\eta_{f d}}{G R}
\end{aligned}
$$

\subsection{Electric Motor Model}

A $150 \mathrm{~kW}$ permanent magnetic motor was applied in this study. An efficient map of the motor was reproduced from Autonomie simulation software. In simulation, the motor efficiency can be obtained from a 2D look-up table through the efficiency curve shown in Figure 2, based on the motor torque and speed.

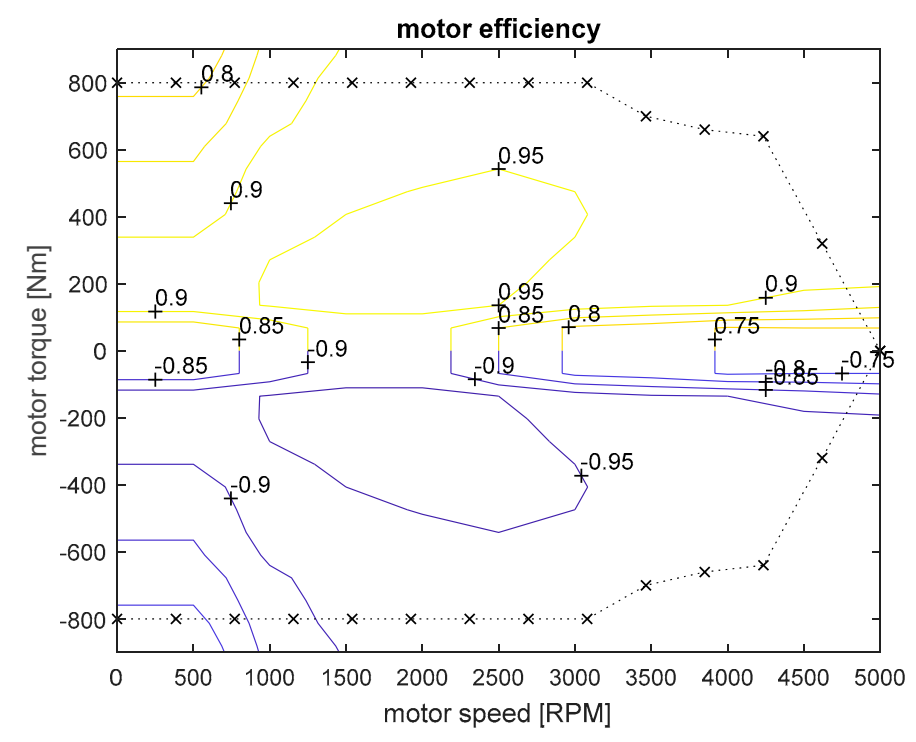

Figure 2. Efficiency of electric motor. 


\subsection{Variable Hydraulic Motor/Pump Model}

Axial slope plate plunger type of hydraulic motor/pump is applied in this study, and its efficiency is obtained through a look-up table, as shown in Figure 3. The fluid flow rate $\left(Q_{P / M}\right)$ and output torque $\left(T_{P / M}\right)$ are calculated according to Equations (8) and (9), where $D_{P / M}$ is the maximum hydraulic motor displacement, $N$ is the hydraulic motor speed, $S_{p}$ is the plate angular position, $\eta_{v P / M}$ is the volumetric efficiency, $\Delta P_{P / M}$ is the pressure difference at the entry and exit, and $\eta_{t P / M}$ is the mechanical efficiency.

$$
\begin{gathered}
Q_{P / M}=D_{P / M} N S_{p} /\left(1000 \eta_{v P / M}\right) \\
T_{P / M}=\left(S_{P} \Delta \mathrm{P}_{P / M} D_{P / M} \eta_{t P / M}\right) / 63
\end{gathered}
$$

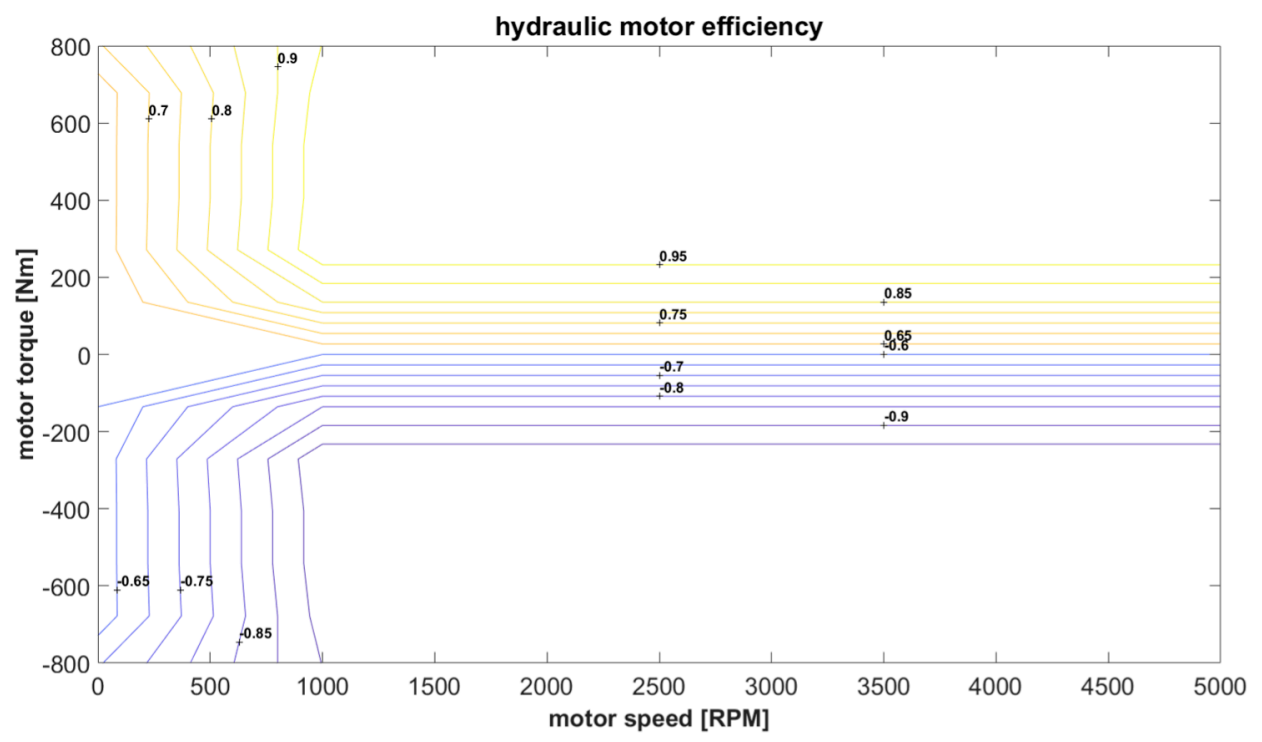

Figure 3. Efficiency of hydraulic motor/pump [1].

\subsection{Battery Model}

The battery used in this study was a lithium ion battery. An RC circuit design was applied, as shown in Equation (10), where $V_{t}$ is the battery terminal voltage, $V_{o c}$ is the battery open-circuit voltage, $I_{b a t}$ is the output current, and $R_{i n t}$ is the internal resistance.

$$
V_{t}=V_{o c}-I_{b a t} \cdot R_{i n t}
$$

Since the terminal voltage and the current can be measured, output of battery power $P_{b a t}$ can be received from Equation (11).

$$
P_{b a t}=I_{b a t} \cdot V_{o c}
$$

Equation (12) can be obtained by substituting Equation (10) to Equation (11).

$$
I_{b a t}=\frac{V_{t}-\left(V_{t}^{2}-4 \cdot R_{i n t} \cdot P_{b a t}\right)^{0.5}}{2 R_{i n t}}
$$

The battery $S O C$ is expressed by the capacity ampere hour. Since $S O C$ changes with the current charging and discharging, $S O C$ can be obtained from Equation (13), where $S O C_{i n t}$ is the initial value of the battery.

$$
S O C=S O C_{i n t}-\frac{\int_{0}^{t} I_{b a t} d t}{A h}
$$




\subsection{Accumulator Model}

For the accumulator model, the influence due to temperature change was not considered in this study, so the gas-state change is set to be adiabatic process (rapid change, $n=1.4$ ). The relationship between the pressure and volume is shown in Equations (14) and (15), during actual expansion and compression of gas.

$$
P V^{n}=C
$$

where $P$ is pressure, $V$ is volume of container area, and $C$ is a constant value.

$$
P_{0} V_{0}^{n}=P_{1} V_{1}^{n}=P_{2} V_{2}^{n}=C
$$

where $P_{0}$ is initial pressure, $P_{1}$ is the maximum activate pressure of accumulator, $P_{2}$ is the minimum activate pressure of accumulator, $V_{0}$ is the total volume of accumulator, $V_{1}$ is the volume of air in accumulator when the pressure is $P_{1}$, and $V_{2}$ is the volume of air in accumulator when the pressure is $P_{2}$.

The boundary movement work, $W_{b}$, of the accumulator can be expressed by Equation (16).

$$
W_{b}=\int_{1}^{2} P d V=P_{1} V_{1} \ln \frac{P_{1}}{P_{2}}
$$

The inlet/outlet fluid, $V_{f}$, of the accumulator can be expressed by Equation (17).

$$
\begin{gathered}
V_{f}=V_{1^{\prime}}-V_{2}{ }^{\prime} \\
=\left(V_{0}-V_{1}\right)-\left(V_{0}-V_{2}\right)=V_{2}-V_{1}=P_{0}^{\frac{1}{n}} V_{0}\left\{\left(\frac{1}{P_{2}}\right)^{\frac{1}{n}}-\left(\frac{1}{P_{1}}\right)^{\frac{1}{n}}\right\}=\left(\frac{P_{0}}{P_{1}}\right)^{\frac{1}{n}} V_{0}\left\{\left(\frac{P_{1}}{P_{2}}\right)^{\frac{1}{n}}-1\right\}
\end{gathered}
$$

The accumulator $S O C$ is expressed by the volume. Since the $S O C$ changes with the volume flow rate, the accumulator $S O C$ can be expressed by Equation (18).

$$
S O C=S O C_{i n t}-\frac{\int_{0}^{t} Q d t}{V_{f}}
$$

\subsection{Vehicle Configurations}

Two vehicles configurations were applied in this study for energy-efficiency comparison. The electric vehicle (EV) configuration is shown in Figure 4, and the HEHV configuration is presented in Figure 5.
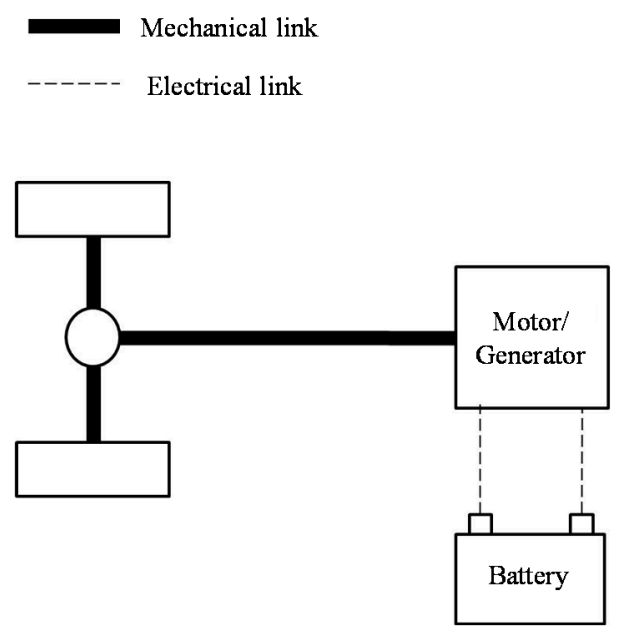

Figure 4. Electric vehicle (EV) configuration. 

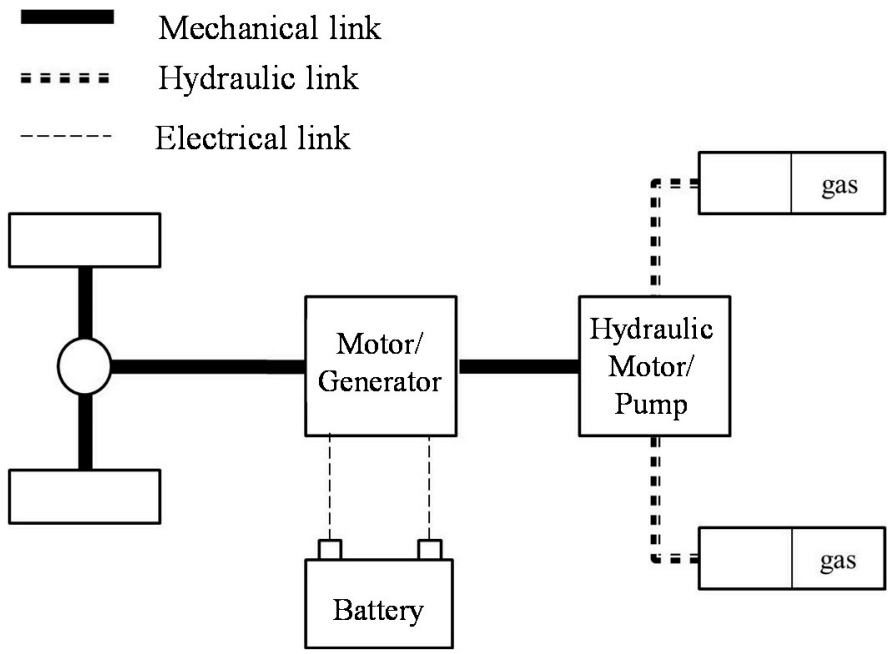

Figure 5. Hydraulic electric hybrid vehicle (HEHV) configuration.

\section{Optimization Control Strategy}

In this study, genetic algorithm (GA) was applied as the optimization function. The rule-based control was taken as the energy management strategy of HEHV first, and the simulation result was compared with the pure-electric-vehicle model. Then, the selected optimization approach was implemented for global optimization. From those results, together with a rule-based control approach, the optimal electricity economic performance was obtained.

The global optimization calculation was made by genetic algorithm. The objective of GA optimization was to minimize electricity consumption, and the objective function was set to be the reciprocal of the lithium ion battery's state of charge, SOL Li, as shown in Equation (19). The setting of objective function in GA could correspond to the fitness function, as shown in Equation (20). Parameters of GA set in this study are shown in Table 1.

$$
\begin{gathered}
\operatorname{cost}=\min \left(1 / \sum(S O C \mathrm{Li})\right) \\
\text { Fitness }=1 / \text { cost }
\end{gathered}
$$

Table 1. Parameter settings of genetic algorithm (GA).

\begin{tabular}{cc}
\hline Gene Length & 20 Bits (10 Bits for Both Acceleration and Accumulator SOC) \\
\hline Group number & 50 \\
Algebra & 40 \\
Mating rate & 0.9 \\
Mutation probability & 0.01 \\
\hline
\end{tabular}

Two design variables (vehicle acceleration and accumulator SOC) were applied to judge the time to use the hydraulic system in control strategy. The thresholds of vehicle acceleration and accumulator $S O C$ were set as selected variables $x$ and $y$ for optimization, respectively. If the vehicle acceleration was higher than the acceleration threshold and the accumulator $S O C$ was higher than the accumulator threshold, the hydraulic pump provided the required power for vehicle acceleration. If the vehicle acceleration was lower than the acceleration threshold and the accumulator SOC was lower than the accumulator threshold, the electric motor provided the required acceleration power. If the vehicle acceleration was higher than the acceleration threshold and the accumulator SOC was lower than the accumulator threshold, the electric motor provided the major portion of required acceleration power. Some other detailed judgements of applying hydraulic pump and the overall control flow are shown 
in Figure 6. To prevent the calculation of variables $x$ and $y$ from exceeding the maximum component scope, the setting constraints of the variables are shown in the constraint Equations (21) and (22).

$$
\begin{gathered}
0<x \leq 1 \text { (vehicle acceleration constraint) } \\
0 \leq y \leq 0.4(\text { accumulator constraint) }
\end{gathered}
$$

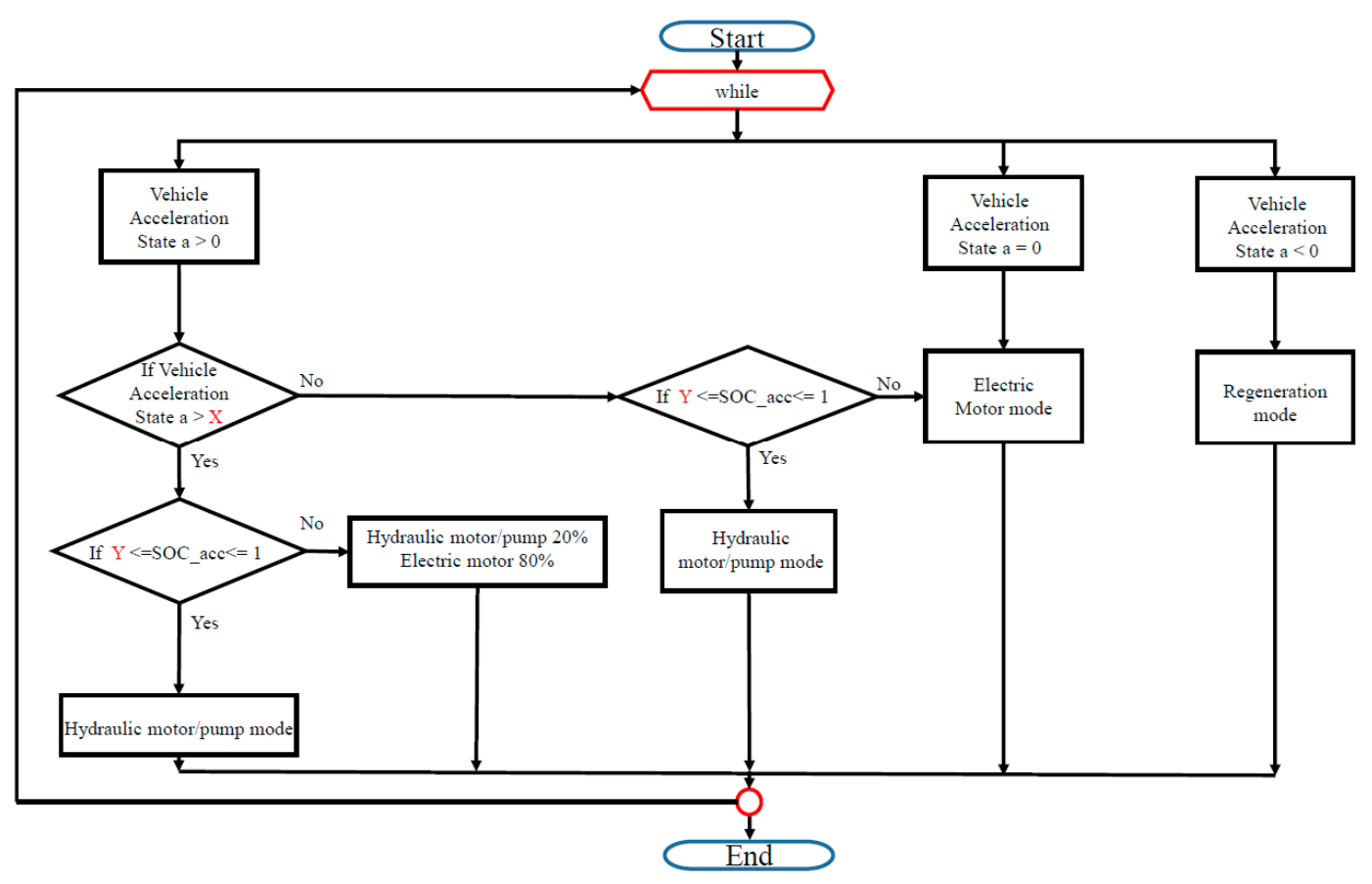

Figure 6. Control flow of genetic algorithm.

The fitness function was adapted to judge whether the solution of GA was suitable for the overall response of the hydraulic system. Values of acceleration threshold $x$ and accumulator threshold $y$ were recorded each time the GA was simulated. After the algorithm completed the iteration set of simulation, its fitness performance was looked up to ensure the value of fitness function was reasonable. The number of mutations and whether the optimization was convergence were checked during the operation of GA. In this study, the convergence of GA was judged by the difference of fitness values between the final four generations. If each difference was smaller than $1 \%$, the optimization reached the convergence. From the solution of optimal fitness value, the recorded variables $x$ and $y$ were selected as the optimal set threshold. This set of variables could be implemented in rule-based control algorithm for real-time simulation and improve the energy consumption. With the implement of genetic algorithm, the rule-based control algorithm for real-time simulation could achieve the energy performance close to optimization.

The thresholds of vehicle acceleration and accumulator SOC calculated from the genetic algorithm were 0.9 and 0.1 , respectively. The diagram of control strategy was modified, as shown in Figure 7. 


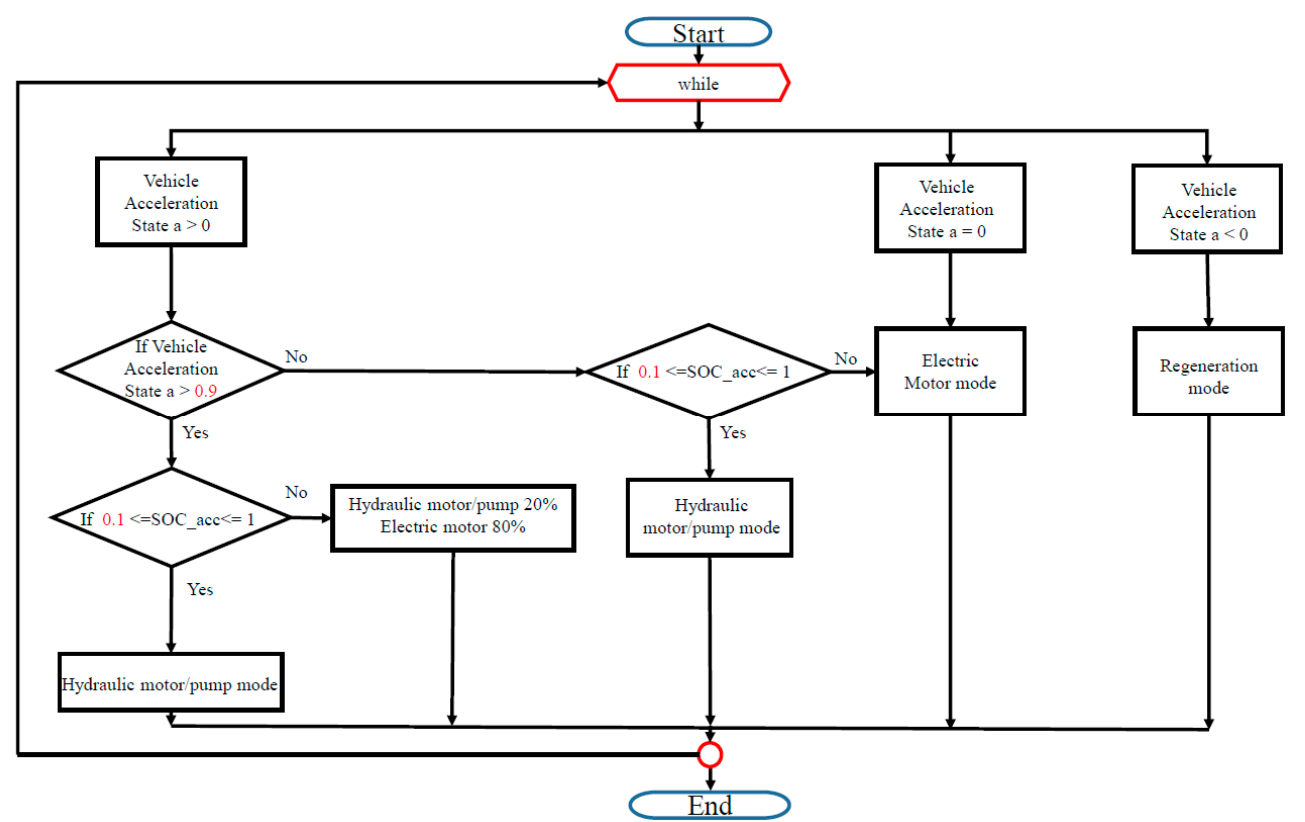

Figure 7. Updated control strategy from genetic algorithm.

\section{Results}

The vehicle parameters of the simulated vehicle are presented in Table 2. The mass of vehicle includes the gross weight, which is $7200 \mathrm{~kg}$, and 20 passengers, which is $1600 \mathrm{~kg}$.

Table 2. Vehicle parameters.

\begin{tabular}{cccc}
\hline Parameter & Symbol & Unit & Value \\
\hline Vehicle mass & $W$ & $\mathrm{~kg}$ & 8800 \\
Wheelbase & & $\mathrm{cm}$ & 378 \\
Wheel track & & $\mathrm{cm}$ & 168 \\
Front area & $A_{f}$ & $\mathrm{~m}^{2}$ & 5.4 \\
Aerodynamic drag coefficient & $C_{D}$ & & 0.28 \\
Rolling resistance coefficient & $\mu_{r}$ & & 0.008 \\
Tire radius & $\mathrm{r}$ & $\mathrm{m}$ & 0.334 \\
Air density & $\rho$ & $\mathrm{kg} / \mathrm{m}^{3}$ & 1.225 \\
Gravitational acceleration & $g$ & $\mathrm{~m} / \mathrm{s}^{2}$ & 9.81 \\
Final reduction gear ratio & $G R$ & & 11.5 \\
Hydraulic system weight & & $\mathrm{kg}$ & 200 \\
\hline
\end{tabular}

In this section, simulation results of the pure electric vehicle and HEHV are compared, and that of the HEHV with optimized energy management strategy is explored. With the energy consumption of the NYCC driving cycle as the analysis basis, the difference of component performance is discussed. Firstly, the pure electric vehicle was established based on the set subsystem model, and it was taken as the basic model. Then the HEHV model was established based on the hydraulic components (hydraulic motor/pump and accumulator), and the rule-based control strategy was applied for the energy management of the power system. Finally, the rule-based control strategy was improved based on the results from the genetic algorithm, to get the HEHV with optimization energy management strategy.

\subsection{EV vs. HEHV (Rule-Based)}

This section compares the difference between the EV and HEHV and presents the causes of the differences. The operating points of the EV electric motor are presented in Figure 8, and those of HEHV electric motor are show in Figure 9. It is obvious that the HEHV electric motor does not work at 
heavy load and low speed, so it was replaced with a hydraulic motor/pump. Therefore, the operating efficiency points concentrate on the high-efficiency region, and the HEHV features better electricity economic performance than the pure electric vehicle.

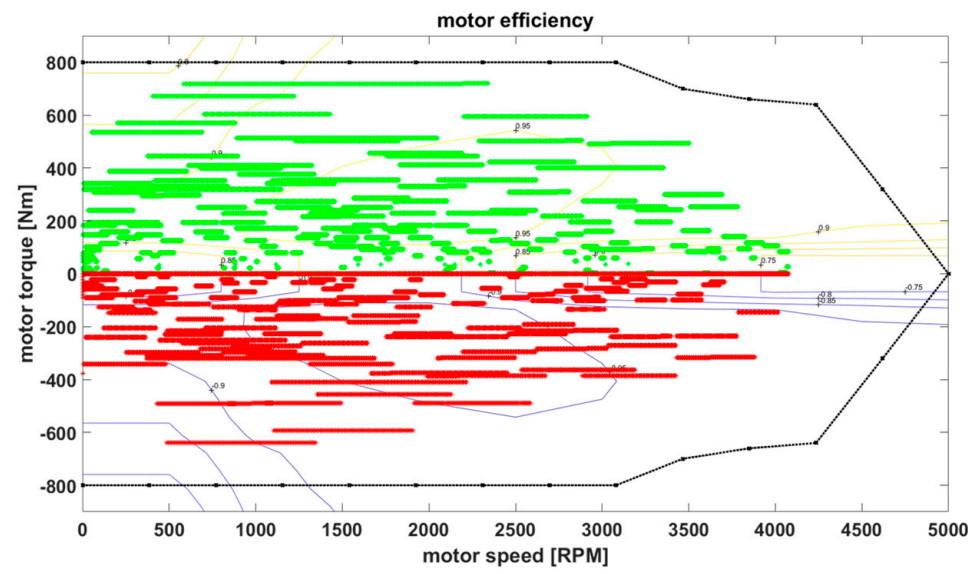

Figure 8. Electric-motor operating points of EV.

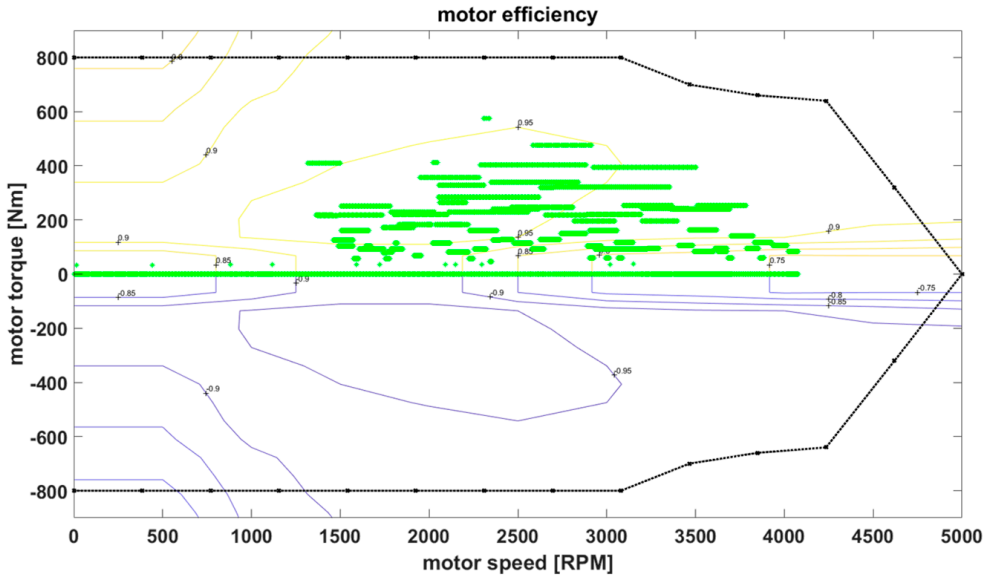

Figure 9. Electric motor operating points of HEHV (rule-based).

In order to better understand the reason that the HEHV has a better economic performance than the pure electric vehicle, the power of electric motors is compared. As shown in Figure 10, the power of HEHV electric motor is smaller than that of pure electric vehicle. Figure 11 shows the comparison of battery SOC, where the electricity economic performance of HEHV is greatly improved.

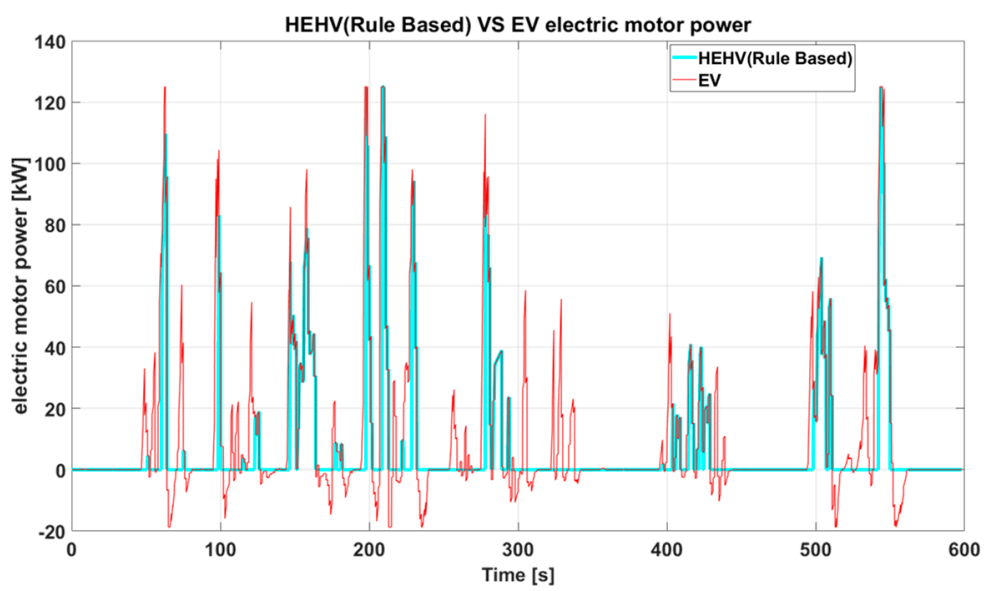

Figure 10. Electric motor power comparison of EV and HEHV. 


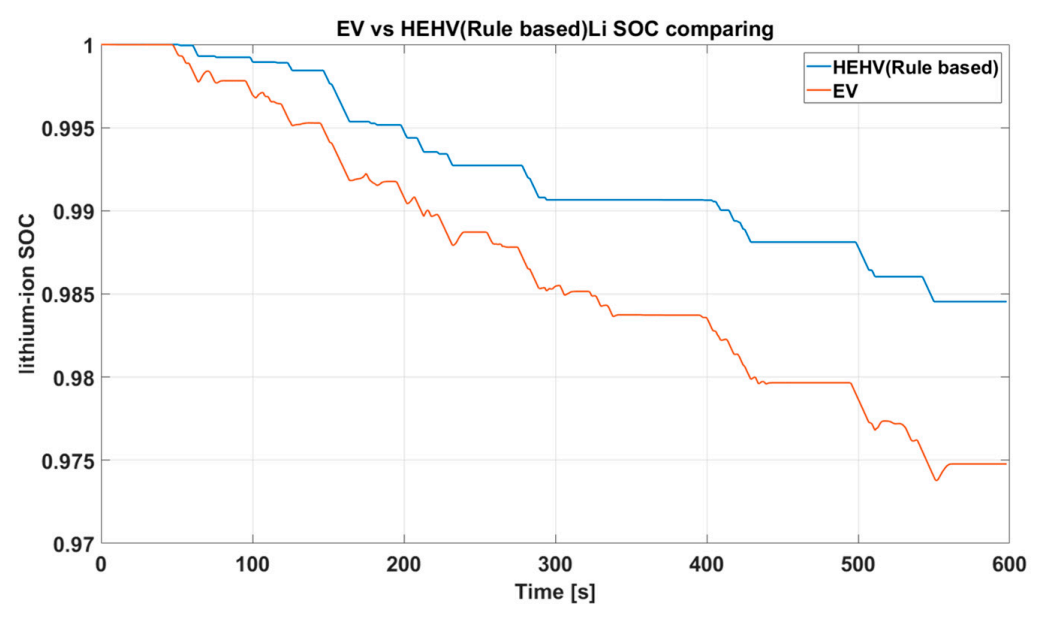

Figure 11. Battery SOC comparison of EV and HEHV.

Table 3 shows the electricity economic performance of the EV and HEHV (rule-based). Since a hydraulic motor functions as the drive at the HEHV's low speed, the use of an electric motor in the low-efficiency zone is reduced, and the electricity is optimized. The electricity economy of the HEHV has $36.5 \%$ improvement over that of EV.

Table 3. Comparison of electricity economic performance between the EV and HEHV (rule-based).

\begin{tabular}{ccc}
\hline & Energy Consumption (kWh) & Electricity Economy (kWh / km) \\
\hline Electric Vehicle (EV) & 0.63 & 0.334 \\
Hydraulic Electric Hybrid Vehicle, & 0.40 & 0.212 \\
HEHV (Rule-Based) & $+36.5 \%$ & $+36.5 \%$ \\
Percent difference &
\end{tabular}

\subsection{HEHV (Rule-Based) vs. HEHV (GA)}

This research had taken the genetic algorithm (GA), together with rule-based control, to perform global optimization, and it got the optimal electricity economic performance. In this section, the HEHV with original rule-based control is compared with the HEHV with modified rule-based control based on the genetic algorithm optimization. The distribution of operating points of the HEHV (rule-based) and HEHV (GA) electric motors is presented in Figures 12 and 13, respectively. The distribution suggests that the operating points of the electric motor after being modified for optimization concentrate more on the high-efficiency region.

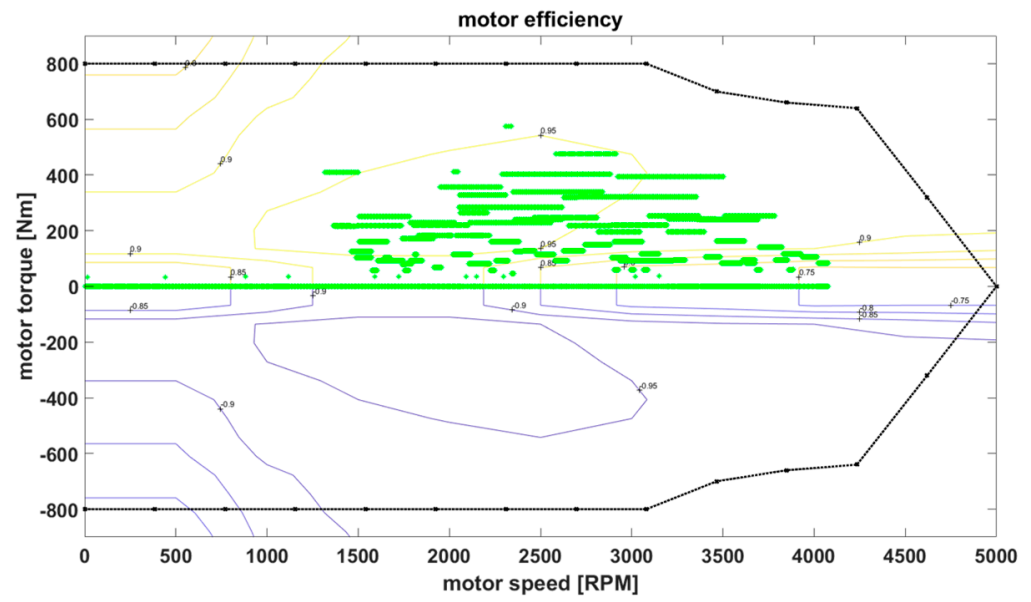

Figure 12. Efficiency points of the HEHV (rule-based) electric motor. 


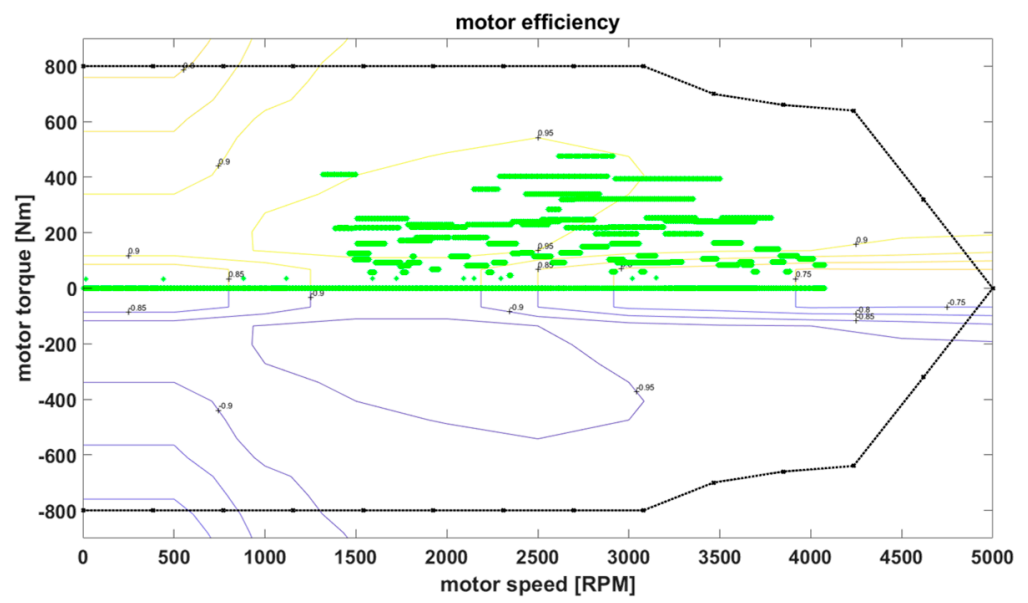

Figure 13. Efficiency points of the HEHV (GA) electric motor.

To understand the motor-use state, the power is compared in this paper, as shown in Figure 14. The usage rate of the electric motor after optimization is less than the original rule-based control strategy, so that better electricity economic performance is reached.

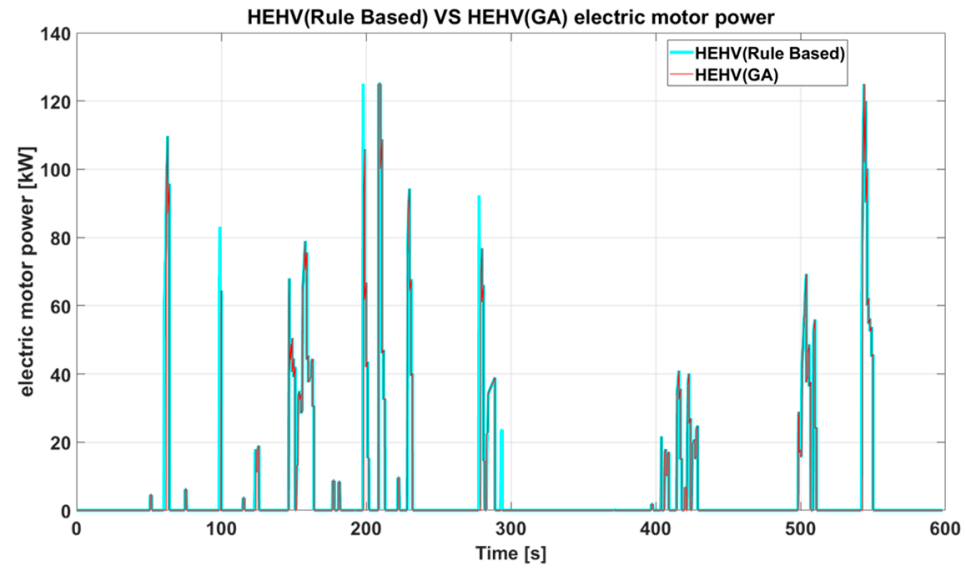

Figure 14. Power comparison between the HEHV (rule-based) and HEHV (GA) electric motors.

The reason why the electric-motor-usage rate after optimization is less can be explained with the help of a comparison of hydraulic motor power, as shown in Figure 15. It is found that the HEHV after optimization uses more hydraulic energy than the original control strategy.

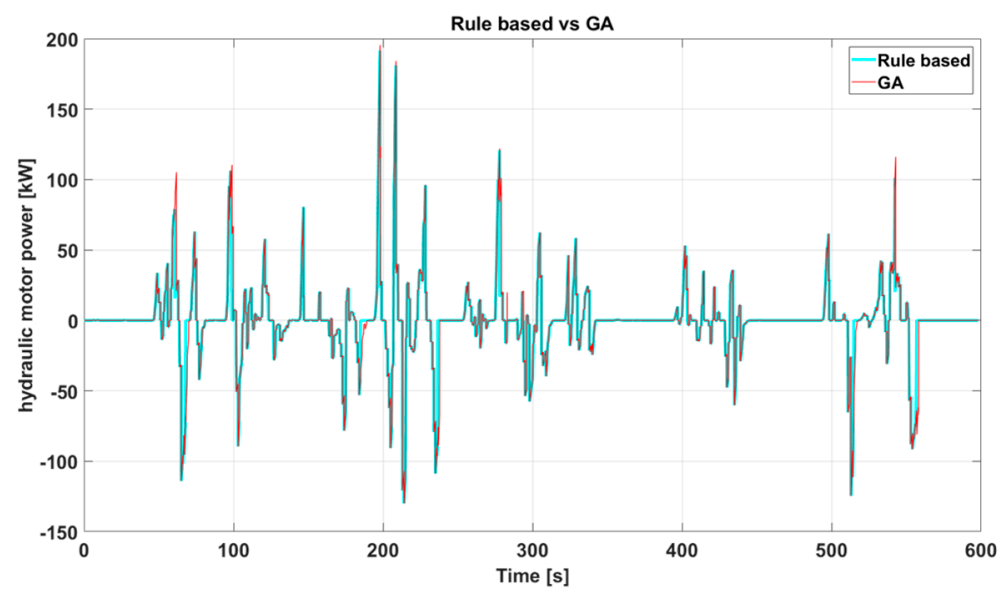

Figure 15. Comparison between HEHV (rule-based) and HEHV (GA) hydraulic motor power. 
As suggested by the comparison of operating-point distribution of hydraulic motor/pump of two control strategies (Figure 16) and state of accumulator use (Figure 17), there are more operating points for the hydraulic motor/pump after optimization than for the original strategy, and they tend to be in the high-efficiency zone. The accumulator is applied more completely due to the wider range of applications for the hydraulic motor/pump.

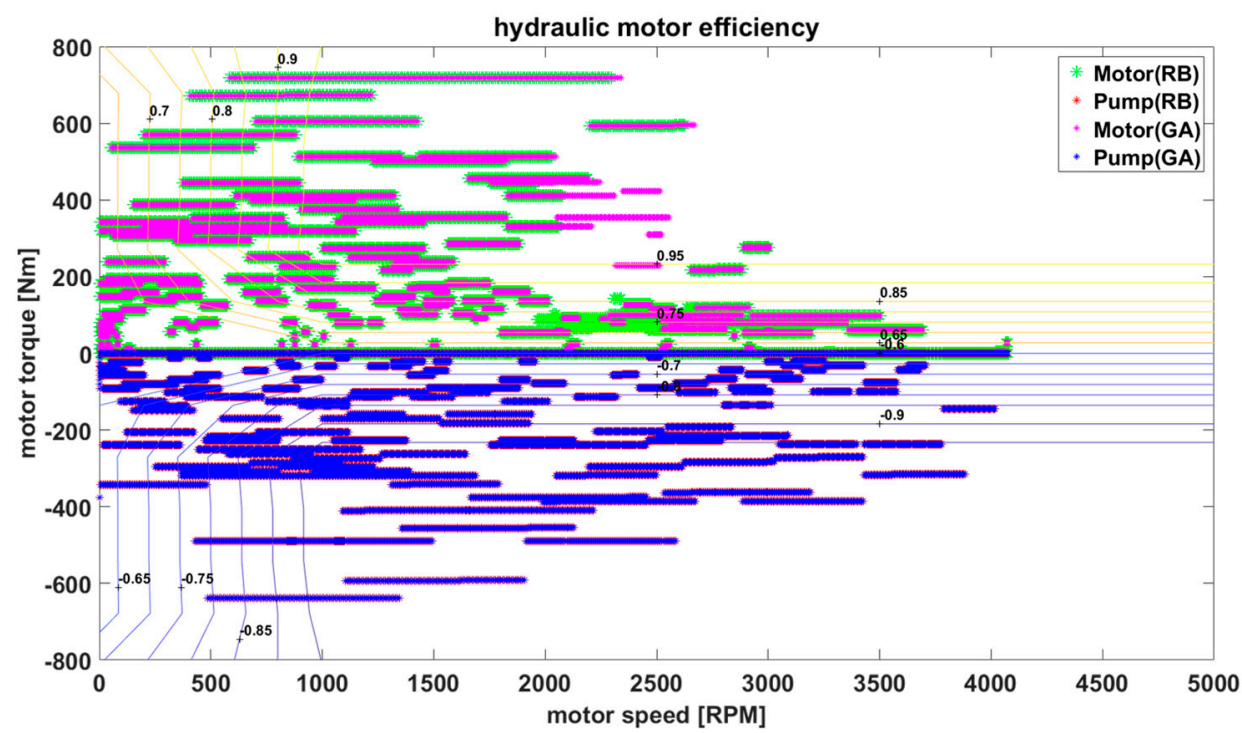

Figure 16. Comparison of operating-point distribution of hydraulic motor/pump (GA vs. rule-based).

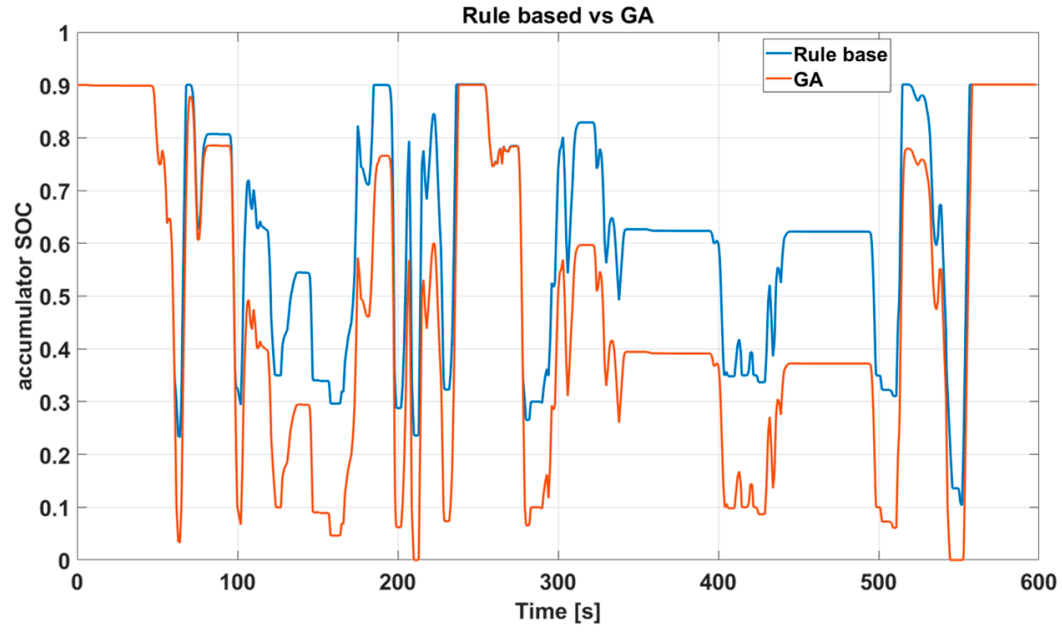

Figure 17. Comparison between HEHV (rule-based) and HEHV (GA) accumulator SOC.

The analysis above indicates that the electricity economic performance of the HEHV after optimization is more improved. Figure 18 shows the battery SOC comparison of the EV and HEHV (Rule based) and HEHV (GA). It is clear that the HEHV after optimization is more improved than the HEHV with original strategy.

The electricity economic performance of the HEHV (rule-based) and HEHV (GA) is drawn in Table 4. Table 5 shows the percentage improvement of electricity in this study. The HEHV with original rule-based control shows $36.5 \%$ improvement over the EV, and the HEHV with modified rule-based control has $43.7 \%$ improvement. 


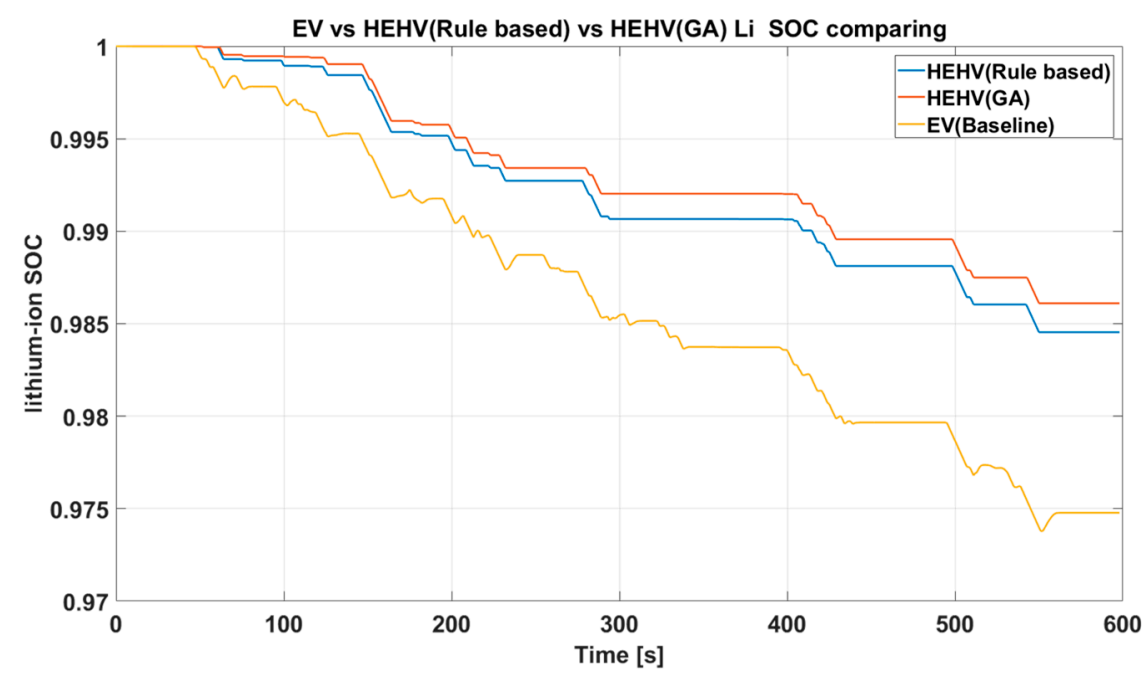

Figure 18. Battery SOC comparison between HEHV (Rule based), HEHV (GA), and EV.

Table 4. Comparison between electricity economic performances.

\begin{tabular}{ccc}
\hline & Power Consumption (kWh) & Electricity Economy (kWh/km) \\
\hline $\begin{array}{c}\text { Hydraulic Electric Hybrid Vehicle, } \\
\text { HEHV (Rule-Based) }\end{array}$ & 0.40 & 0.212 \\
Hydraulic Electric Hybrid Vehicle, & 0.355 & 0.188 \\
$\begin{array}{c}\text { HEHV (GA) } \\
\text { Percent difference }\end{array}$ & $+11.3 \%$ & $+11.4 \%$ \\
\hline
\end{tabular}

Table 5. Electricity improvement percentage.

\begin{tabular}{cc}
\hline & Percent of Improvement \\
\hline Electric Vehicle (EV) & - \\
Hydraulic Electric Hybrid Vehicle, HEHV (Rule-Based) & $36.5 \%$ \\
Hydraulic Electric Hybrid Vehicle, HEHV (GA) & $43.7 \%$ \\
\hline
\end{tabular}

\section{Discussion}

This research mainly targeted the medium and large trucks for energy efficiency, and a hydraulic hybrid electric powertrain system was proposed to apply on the medium duty vehicle for energy efficiency. SimuLink simulation models of the EV and HHEV were built to evaluate the efficiency of the HHEV system. Compared to the EV system, the HHEV system had better energy efficiency, but the control algorithm was not optimized. To improve the efficiency, the genetic algorithm was implemented to achieve the optimized energy efficiency. Since GA was a global optimization algorithm which required longer CPU time for calculation and was not suitable for real-time control, the result of design variables of GA was required to apply on the real-time control strategy, rule-based control. In this research, two design variables of GA, thresholds of vehicle acceleration and hydraulic accumulator SOC, were optimized. These two optimized variables were applied in the HHEV simulation. From the simulation result, Tables 4 and 5, the rule-based model with GA could further improve the energy efficiency. The simulation results show that the electricity economic performance of the designed hydraulic hybrid vehicle was improved by $36.5 \%$ when compared to that of pure electric vehicle. The performance of energy consumption after genetic algorithm optimization was improved by $43.7 \%$.

\section{Conclusions}

In this study, HEHV energy management strategy was applied, and Matlab/Simulink simulation program was utilized to establish a backward simulation model, to simulate the large-vehicle energy 
consumption. Movement situation of power components, SOC of energy storage components, and overall electricity economic performance of the pure electric vehicle and HEHV were obtained with the NYCC driving cycle. The following results can be achieved after simulation in this study:

- In the performance analysis of the pure electric vehicle, the electricity consumption of the set driving cycle is $0.334 \mathrm{kWh} / \mathrm{km}$, and this is taken as the basic model for future comparison.

- In HEHV analysis (rule-based control), the electricity economic performance after simulation of the set driving cycle is $0.212 \mathrm{kWh} / \mathrm{km}$, which is greatly improved than $0.334 \mathrm{kWh} / \mathrm{km}$ of pure electric vehicle, saving $36.5 \%$ of electricity. This is mainly because the hydraulic motor/pump in the pumping mode (energy recovery state) is more able to absorb, recover, and store the vehicle kinetic energy than electric motor, and the hydraulic motor/pump also avoids the application of electric motor at low speed.

- In the HEHV optimization analysis (genetic algorithm), $11.3 \%$ and $43.7 \%$ of electricity can be saved as compared with the HEHV (rule-based control) and pure electric vehicle, respectively.

- Through the HHEV simulation, the genetic algorithm was able to improve the energy efficiency of the HHEV by adjusting the chosen design variables of control strategy.

Author Contributions: Conceptualization, T.-S.L.; investigation, T.-S.L.; methodology, H.-Y.H.; project administration, J.-S.C.; software, J.-S.C.; validation, H.-Y.H. and J.-S.C. All authors have read and agreed to the published version of the manuscript.

Funding: This research received no external funding.

Conflicts of Interest: The authors declare no conflict of interest.

\section{Nomenclature}

$A_{f} \quad$ front area of the vehicle

Ah battery capacity (ampere hour)

$C_{D} \quad$ aerodynamic resistance coefficient

$D_{P / M} \quad$ maximum hydraulic motor displacement

$F_{t} \quad$ vehicle tractive effort

$F_{\text {tire }} \quad$ tyre force

GR final transmission gear ratio

$I_{\text {bat }} \quad$ battery output current

$N \quad$ hydraulic motor speed

$P \quad$ accumulator pressure

$P_{b a t} \quad$ battery power

$Q_{P / M} \quad$ hydraulic pump fluid flow rate

$R_{a} \quad$ aerodynamic resistance

$R_{c} \quad$ grading resistance

$R_{\text {int }} \quad$ battery internal resistance

$R_{r} \quad$ rolling resistance

$R_{r, A} \quad$ front wheel rolling resistance

$R_{r, B} \quad$ rear wheel rolling resistance

$R_{S} \quad$ acceleration resistance

$S_{p} \quad$ hydraulic pump plate angular position

SOC state of charge

$S O C_{\text {int }}$ initial value battery state of charge

SOL Li lithium ion battery SOC

$T_{\text {drive }}$ tyre torque

$T_{P / M} \quad$ hydraulic pump torque

$V \quad$ volume of accumulator container area

$V_{f} \quad$ accumulator inlet/outlet fluid 
$V_{o c} \quad$ battery open circuit voltage

$V_{t} \quad$ battery terminal voltage

$W \quad$ vehicle weight

$W_{b} \quad$ accumulator boundary movement work

a vehicle acceleration

$g \quad$ gravity acceleration

$r \quad$ tyre radius

$v_{w} \quad$ wind speed

$\eta_{f d} \quad$ transmission efficiency

$\eta_{t P / M} \quad$ hydraulic pump mechanical efficiency

$\eta_{v P / M} \quad$ hydraulic pump volumetric efficiency

$\theta \quad$ road slope angle

$\mu_{r} \quad$ rolling resistance coefficient

$\rho \quad$ air density

$\omega_{\text {drive }} \quad$ tyre angular speed

$\Delta P_{P / M} \quad$ hydraulic pump pressure difference at the entry and exit

\section{References}

1. Sokar, M. Investigation of Hydraulic Transmissions for Passenger Cars. Ph.D. Dissertation, Aachen University, Aachen, Germany, February 2011.

2. Chen, J.S. Energy efficiency comparison between hydraulic hybrid and hybrid electric vehicles. Energies 2015, 8, 4697-4723. [CrossRef]

3. Ramakrishnan, R.; Hiremath, S.S.; Singaperumal, M. Theoretical investigations on the effect of system parameters in series hydraulic hybrid system with hydrostatic regenerative braking. J. Mech. Sci. Technol. 2012, 26, 1321-1331. [CrossRef]

4. Salmasi, F.R. Control strategies for hybrid electric vehicles: Evolution, classification, comparison, and future trends. IEEE Trans. Veh. Technol. 2007, 56, 2393-2404. [CrossRef]

5. Lu, X.; Chen, Y.; Wang, H. Multi-objective optimization based real-time control for PEV hybrid energy management systems. In Proceedings of the IEEE Applied Power Electronics Conference and Exposition (APEC), San Antonio, TX, USA, 4-8 March 2018; pp. 969-975.

6. Zeng, Y.; Cai, Y.; Kou, G.; Gao, W.; Qin, D. Energy management for plug-in hybrid electric vehicle based on adaptive simplified-ECMS. Sustainability 2018, 10, 2060. [CrossRef]

7. Wang, X.; He, H.; Sun, F.; Zhang, J. Application study on the dynamic programming algorithm for energy management of plug-in hybrid electric vehicles. Energies 2015, 8, 3225-3244. [CrossRef]

8. Yu, J.; Liu, N.; Zhang, Y.; Wang, B. Modeling and control strategy simulation of extended-range electric vehicle. In Proceedings of the 2011 International Conference on Transportation, Mechanical, and Electrical Engineering (TMEE), Changchun, China, 16-18 December 2011; pp. 829-832.

9. Gao, J.P.; Zhu, G.M.G.; Strangas, E.G.; Sun, F.C. Equivalent fuel consumption optimal control of a series hybrid electric vehicle. Proc. Inst. Mech. Eng. Part D J. Automob. Eng. 2009, 223, 1003-1018. [CrossRef]

10. Konev, A.; Lezhnev, L.; Kolmanovsky, I. Control Strategy Optimization for a Series Hybrid Vehicle; SAE Paper No. 2006-01-0663; SAE World Congress: Detroit, MI, USA, 2006.

11. Liu, X.; Fan, Q.; Zheng, K.; Duan, J.; Wang, Y. Constant SOC control of a series hybrid electric vehicle with long driving range. In Proceedings of the IEEE International Conference on Information and Automation, Harbin, China, 11-13 August 2010; pp. 1603-1608.

12. Li, S.G.; Sharkh, S.M.; Walsh, F.C.; Zhang, C.N. Energy and battery management of a plug-in series hybrid electric vehicle using fuzzy logic. ieee trans. Veh. Technol. 2011, 60, 3571-3585. [CrossRef]

13. Ho, P.; Klong, E. Intelligent Energy Distribution for Series HEVs Using Determined Optimal Driving Patterns via a Genetic Algorithm; SAE Paper No. 2013-01-0572; Graduate Faculty of North Carolina State University: Raleigh, NC, USA, 2013.

14. Xu, Q.; Luo, X.; Jiang, X.; Zhao, M. Research on double fuzzy control strategy for parallel hybrid electric vehicle based on GA and DP optimization. IET Electr. Syst. Transp. 2018, 8, 144-151. [CrossRef] 
15. Kaur, J.; Saxena, P.; Gaur, P. Genetic algorithm based speed control of hybrid electric vehicle. In Proceedings of the 2013 Sixth International Conference on Contemporary Computing, Noida, India, 8 August 2013; pp. 65-69.

16. Hu, F.; Zhao, Z. Optimization of control parameters in parallel hybrid electric vehicles using a hybrid genetic algorithm. In Proceedings of the 2010 IEEE Vehicle Power and Propulsion Conference, Lille, France, 1-3 September 2010; pp. 1-6.

(C) 2020 by the authors. Licensee MDPI, Basel, Switzerland. This article is an open access article distributed under the terms and conditions of the Creative Commons Attribution (CC BY) license (http://creativecommons.org/licenses/by/4.0/). 